\title{
Subtle morphological changes in the mandible of Tabby mice revealed by micro-CT imaging and elliptical Fourier quantification
} \author{
François Clauss ${ }^{1,2}$, Hervé Lesot ${ }^{1}$, André Constantinesco ${ }^{3,4}$ and Matthieu Schmittbuhl1,2* \\ Institut National de la Santé et de la Recherche Médicale, INSERM UMR 977, University of Strasbourg, Strasbourg, France \\ 2 Faculty of Dentistry, University of Strasbourg, Strasbourg, France \\ ${ }^{3}$ Service de Biophysique et Médecine Nucléaire, Strasbourg University Hospital, Strasbourg, France \\ 4 Faculty of Medicine, University of Strasbourg, Strasbourg, France
}

Fabien Bornert ${ }^{1,2}$, Philippe Choquet ${ }^{3,4}$, Catherine I. Gros ${ }^{1,2}$, Gaelle Aubertin ${ }^{3,4}$, Fabienne Perrin-Schmitt ${ }^{1,4}$,

\section{Edited by:}

Anna Petryk, University of Minnesota

USA

\section{Reviewed by:}

Yiping Chen, Tulane University, USA

Martyn Cobourne, King's College

London, UK

Thimios Mitsiadis, University of Zurich,

Switzerland

Peter Hammond, University College

London, UK

\section{*Correspondence:}

Matthieu Schmittbuhl, Faculty of

Dentistry, University of Strasbourg, 1

Place de I'Hôpital, F-67000 Strasbourg,

France.

e-mail:m.schmittbuhl@unistra.fr
X-linked hypohidrotic ectodermal dysplasia (XLHED) is a genetic disorder due to a mutation of the EDA gene and is mainly characterized by an impaired formation of hair, teeth and sweat glands, and craniofacial dysmorphologies. Although tooth abnormalities in Tabby (Ta) mutant mice - the murine model of XLHED - have been extensively studied, characterization of the craniofacial complex, and more specifically the mandibular morphology has received less attention. From 3D micro-CT reconstructions of the left mandible, the mandibular outline observed in lateral view, was quantified using 2D elliptical Fourier analysis. Comparisons between Ta specimens and their wild-type controls were carried out showing significant shape differences between mouse strains enabling a clear distinction between hemizygous Ta specimens and the other mouse groups (WT and heterozygous Eda ${ }^{\mathrm{T} / \mathrm{l}+}$ specimens). Morphological differences associated with HED correspond not only to global mandibular features (restrained development of that bone along dorsoventral axis), but also to subtle aspects such as the marked backward projection of the coronoid process or the narrowing of the mandibular condylar neck. These modifications provide for the first time, evidence of a predominant effect of the Ta mutation on the mandibular morphology. These findings parallel the well described abnormalities of jugal tooth row and skeletal defects in Ta mice, and underline the role played by EDA-A in the reciprocal epithelial-mesenchymal interactions that are of critical importance in normal dental and craniofacial development.

Keywords: hypohidrotic ectodermal dysplasia, mandibular dysmorphology, micro-CT, elliptical Fourier analysis, Eda gene

\section{INTRODUCTION}

Hypohidrotic ectodermal dysplasias (HED) are a large and heterogenous group of heritable genodermatoses, encompassing more than 200 distinct syndromes and characterized by structural abnormalities of ectodermal appendages (DiGiovanna et al., 2009; Itin, 2009). Ectodermal dysplasias are not considered as pure one-layer syndromes. Indeed, mesodermal abnormalities are also found given the close reciprocal interactions observed between the ectodermal and mesodermal layers (Itin, 2009). The most prevalent HED is X-linked hypohidrotic ectodermal dysplasia (XLHED) and is due to a mutation in EDA gene (Vincent et al., 2001). EDA gene undergoes alternative splicing and encodes a morphogenetic factor called Ectodysplasin-A1 isoform with tumor necrosis factor (TNF) and collagen domains involved in cell interaction regulation, epithelial morphogenesis, and ectodermal development (Monreal et al., 1998).

The clinical phenotype of individuals affected by HED is complex and includes hypotrichosis, dental agenesis and morphological defects, onychodysplasia and eccrine glands aplasia, or hypoplasia, being the main features (Pinheiro and Freire-Maia, 1994). Craniofacial and anthropometric changes are also described in
XLHED and consist in reduced and retrognathic maxilla, frontal prominence, cranial base modifications, reduced facial convexity and facial height with deficiencies in sagittal and transverse skeletal growth (Johnson et al., 2002; Arslan et al., 2007; Clauss et al., 2008). Global reduction of facial growth, especially in the middle third of the facial skeleton, and mandibular growth retardation are integrated in HED craniofacial phenotype (Dellavia et al., 2010). Bone structural modifications have also been described in patients affected by XLHED and correspond to local increases of alveolar and basal bone densities and hypercorticalization (Lesot et al., 2009). Heterozygous female carriers of XLHED exhibit moderate phenotypic manifestations consisting in hypodontia, mild dental morphological defects, distribution of abnormal skin along Blaschko lines, and some craniofacial changes as midface hypoplasia and abnormal cranial base length (Cambiaghi et al., 2000).

Tabby (Ta) mutant mouse mimicking human XLHED, results from a Ta gene mutation sharing a 97\% sequence homology with EDA human gene (Srivastava et al., 1997). Phenotypically, this mouse model displays the same ectodermal defects with alopecia, eccrine glands hypoplasia, dental ageneses, microdontia or dental crown, and root morphological alterations (Tucker et al., 2000; 
Kristenova et al., 2002; Charles et al., 2009). Dental abnormalities in Ta mice were characterized by a marked phenotypic variability (Kristenova et al., 2002; Peterkova et al., 2002). A skeletal phenotype in Ta mice was also described, consisting of spontaneous tail vertebral fractures linked to epiphyseal and subepiphyseal dysplasia as well as bone microstructural defects with increased tibial trabecular interconnectivity (Hill et al., 2002). Interestingly, there are few observations about craniofacial morphology in Ta mice and no quantitative data describing the mandibular aspects in this syndrome seem to be available in literature.

Given its relatively low prevalence (Pinheiro and Freire-Maia, 1994), studies of humans may never provide enough data to document the extent of phenotypic variation in HED. Furthermore, the underlying assumption is that the mandibular shape phenotype will reflect main and direct morphological effects of the EDA gene mutation associated to HED. Consequently, the study of the corresponding mutant mouse model provides a pertinent comparative framework that is likely to highlight the genotype-phenotype correlation in HED, offering further information on the role of EDA gene in the etiology of mandibular dysmorphology in HED.

In the current study, we document the morphological changes in the mandible of Ta mice in order to complete the general phenotypic picture of HED syndrome. To accomplish it, 2D elliptical Fourier analysis (Kuhl and Giardana, 1982; Schmittbuhl et al., 2003) of the whole mandibular outline precisely recorded from microCT 3D reconstruction of the left mandible was carried out. Using such detailed characterization of the mandible, our objectives are to (1) quantitatively evaluate morphological variation among and within the different mice groups under study, (2) depict the shape differences resulting from the phenogenetic processes associated with XLHED, and (3) provide a comparative framework that could be pertinent in interpreting the genotype-phenotype correlation in HED.

\section{MATERIALS AND METHODS TABBY MOUSE MODEL}

Tabby mice harbored the $E d a^{\text {Ta }}$ null allele of the Eda gene, carried by the X-chromosome. The Ta mutation results from a $2 \mathrm{~kb}$ deletion which includes the exon 1 coding region. $E d a^{\text {Ta }}$ mice were generated from the inbred Ta strain B6CBACa $\mathrm{A}^{\mathrm{w}-\mathrm{J}} / \mathrm{A}-E d a^{\mathrm{T} a} / \mathrm{J}-\mathrm{XO}$ (Jackson Laboratory, USA). The phenotype was identified according to external morphological criteria: bald patches behind ears and deformities in the distal portion of the tail for hemizygous males $\left(E d a^{\mathrm{T} / \mathrm{Y}}\right)$, striping of the coat for heterozygous females $\left(E d a^{\mathrm{T} a /+}\right)$. WT mice were generated by inbreeding wild-type animals derived from the B6129PF2/J strain considered as control for strains designated by B6. WT mice and Ta heterozygous females and hemizygous males were genotyped according to previously published protocols (Bourgeois et al., 1998) through PCR amplification using specific forward and reverse oligonucleotides (3F-7R, 6F-8R, and 5F-6R), derived from -3 to $+1.9 \mathrm{~kb}$ murine genomic sequence surrounding exon 1 of the WT Eda gene. The presence of a deletion in Eda gene exon 1 was observed in Ta specimens, as the 6F-8R primers allowed for amplification only in Ta heterozygous females and WT. The expected genotype of each Ta mice was confirmed by the absence of the 5F-6R primed fragments in Ta males. Mice population sample included 74 specimens, 15 hemizygous males
$\left(E d a^{\mathrm{T} / \mathrm{Y}}\right), 24$ heterozygous females $\left(E d a^{\mathrm{Ta} /+}\right)$, and 35 control WT mice (19 males, 16 females). The Ta sample overlapped the sample studied by Kristenova et al. (2002). All the specimens studied were adults (3-month-old mice). Mice were killed by intraperitoneal lethal injection of pentobarbital. The experimental protocol was designed in compliance with recommendations of the EEC (86/609/ CEE) for the care and use of laboratory animals. The animals were fixed in 1:10 37\% formol, 3:4 100\% ethanol, and 3:20 distilled water solution for 15 days.

\section{MICRO-CT SCANNING}

Ex vivo imaging of full heads was performed using a micro-CT system (eXplore CT 120, GE Healthcare, Waukesha, WI, USA). The protocol used acquired 360 views on $360^{\circ}$, at $100 \mathrm{kV}, 50.0 \mathrm{~mA}$, with an exposure time of $20 \mathrm{~ms}$ (Figure 1A). The duration of one acquisition was less than $4 \mathrm{~min}$. A Feldkamp algorithm of backprojection led to the reconstruction of a volume made of cubic voxels of $50 \mu \mathrm{m} \times 50 \mu \mathrm{m} \times 50 \mu \mathrm{m}$.

\section{IMAGE PROCESSING}

The first steps of the image analysis procedure were performed using MicroView ${ }^{\circledR}$ (GE Healthcare, Waukesha, WI, USA). The left hemimandibles were segmented by using region of interest (ROI) and volume of interest (VOI) functions. A 3D virtual reconstruc-
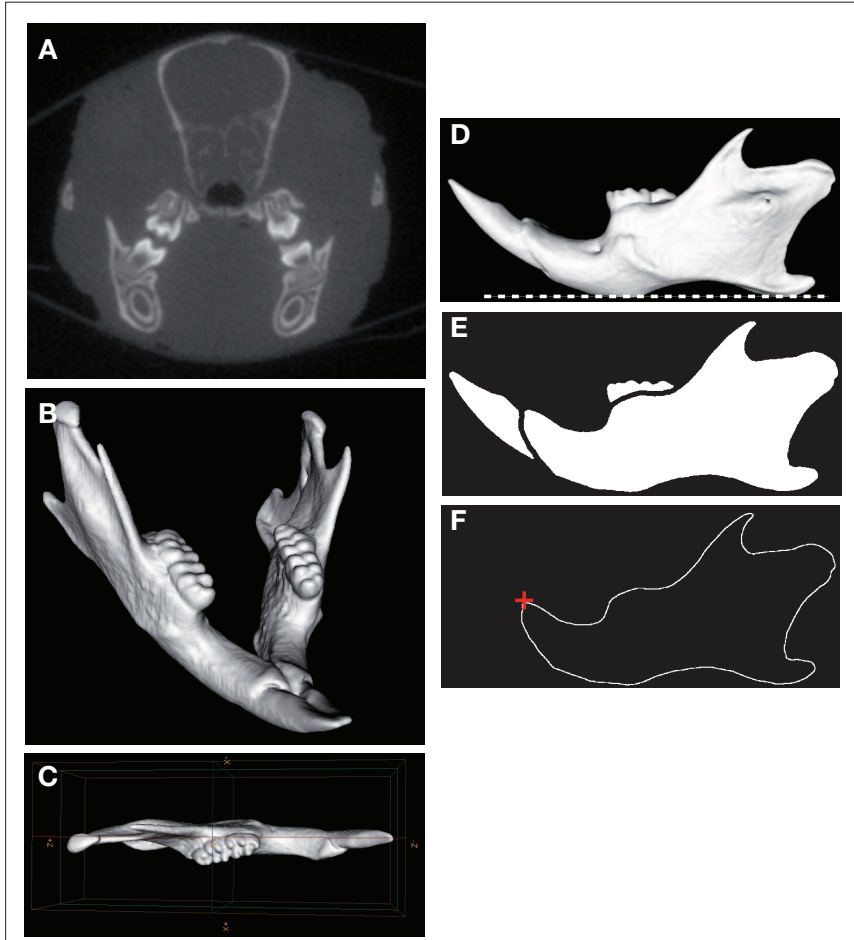

FIGURE 1 | Micro-CT scanning and image processing of the mouse mandible. Transverse micro-CT slice of the craniofacial skeleton (A). Threedimensional reconstruction of the whole mandible (B). The left hemimandible appeared as a relatively plane structure and was aligned parallel with the sagittal plane (C). Horizontal orientation of the mandibular plane (dashed white line) (D). Binarized image and segmentation of the mandibular bone (E). Extraction of the mandibular outline (F); the starting point of the outline (red cross) corresponded to the anterodorsal tip of the incisor alveolar bone. 
tion of each left mandible was then computed using the isosurface volume rendering module with a threshold value set at $600 \mathrm{HU}$ (Figure 1B). The hemimandible was aligned parallel with the sagittal plane (Figure 1C) and the mandibular plane was oriented horizontally (Figure 1D). The mandibular images were then processed using the software package Visilog $5.4^{\circledast}$ (Noesis, Gif sur Yvette, France). As each hemimandible could be considered as a relatively plane bony structure (Figure 1C), the 2D mandibular outline was extracted from the binarized image of each hemimandible lateral view as a series of 512 points expressed by its 2D Cartesian coordinates (Figure 1E). The standardized starting point of the outline corresponded to the anterodorsal tip of the incisor alveolar bone (Figure 1F).

\section{ELLIPTICAL FOURIER ANALYSIS}

Elliptical Fourier analysis was used to describe the mandibular outline following our previous work (Schmittbuhl et al., 2003). The size normalization procedure consisted in a recalculation of the mandibular outline using the same value of the enclosed area for all mandibles. Step-by-step reconstructions of the mandibular outlines were performed using an increasing number of harmonics (Figure 2). A fit index, obtained from the sum of squared distances between the reconstructed points and the corresponding points on the original outline, was calculated for each step of the reconstruction (Schmittbuhl et al., 2003). Reconstructions were made point by point and corresponded to a summation of vectors, each vector having its origin on the center of the corresponding harmonic ellipse and its extremity on the contour of the considered ellipse (Video S1 in Supplementary Material).

\section{MANDIBULAR SIZE VARIATION ANALYSIS}

Size variation was evaluated on the mandibular area corresponding to the enclosed area of the mandible in lateral projection.

\section{STATISTICAL ANALYSES}

Each harmonic corresponded to an ellipse summarized by three elliptical descriptors (Figure 3): major axis length, minor axis length, and orientation of the major axis of the ellipse. The summary statistics (mean, SD) of the elliptical Fourier descriptors were derived for each mouse strains and each sex (WT males, WT females, $\left.E d a^{\mathrm{Ta} / \mathrm{Y}}, E d a^{\mathrm{Ta} /+}\right)$. The quality of fit with the normal distribution was estimated by using the Kolmogorov-Smirnov test. The degree of distinction among mouse strains was assessed and displayed graphically using a discriminant analysis. Correlations between elliptical descriptors and discriminant axes were calculated. The unbiased Mahalanobis $D^{2}$ distances - e.g., corrected for unequal sample sizes - between the centroids of each group were calculated to express inter-mouse strains variability. A classification procedure of the specimens was also performed from the derived discriminant scores, and a cross-validation (Jackknifed classification) was applied to estimate the correct classification rate of the discriminant functions (Everitt and Rabe-Hesketh, 2001). Next, mandibular size comparisons between mouse strains were performed using an analysis of variance (ANOVA). The homogeneity of variance was assessed using Levene's Test. In the presence of unequal variance, a Welch $F$ test was used to detect significant between-group differences. A post hoc pairwise comparison based

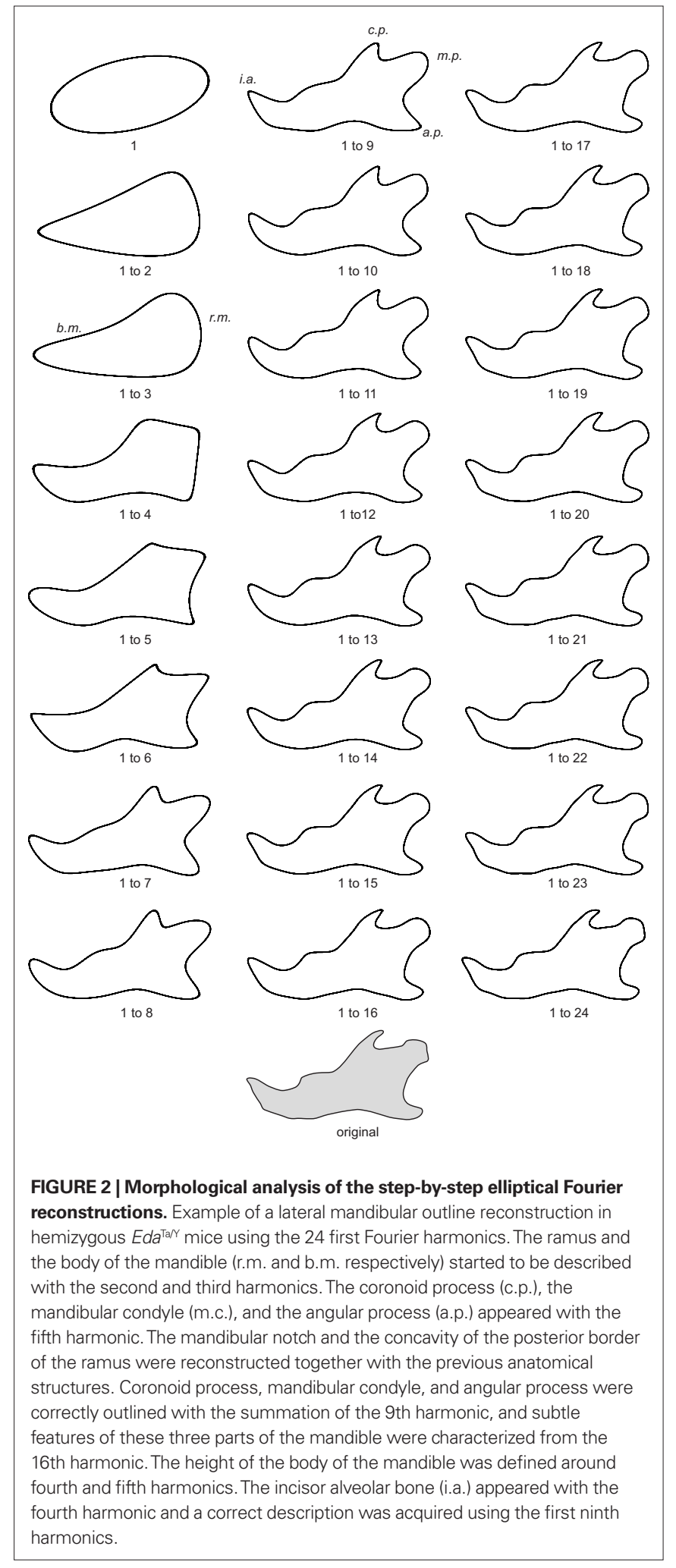

on Tukey's test was then computed to determine which mouse strains were statistically different from the others. All the analyses were performed using Statistica 7.1 software package (Statsoft Inc., Tulsa, OK, USA) and PAST 1.92 (Hammer et al., 2001). 


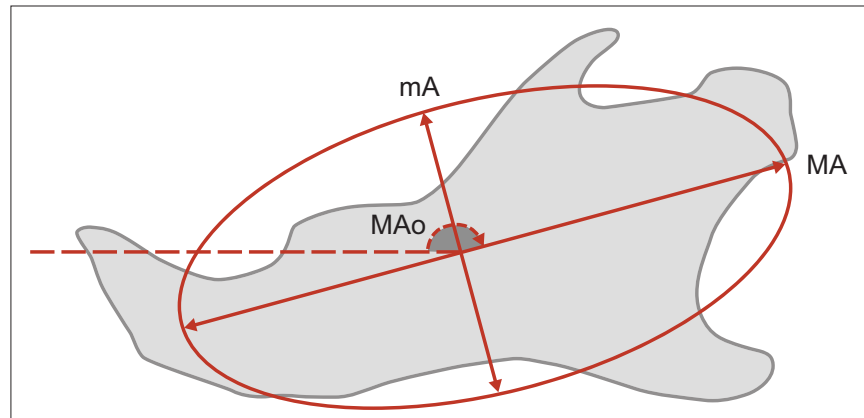

FIGURE 3 | Each harmonic corresponded to an ellipse summarized by three elliptical descriptors: major axis length (MA), minor axis length ( $\mathrm{mA}$ ), and orientation of the major axis of the ellipse (MAO). The

orientation of the ellipse corresponds to the angle formed by the major axis and the horizontal reference axis.

\section{RESULTS}

Statistics for the elliptical Fourier descriptors (major axis length, minor axis length, elliptical orientation) of the mandibular outline for each mouse strain are reported in Table 1. An example of step-by-step reconstruction of the lateral mandibular outline is illustrated for the 24 first Fourier harmonics in Figure 2.

For each individual, stepwise summation of the first 24 elliptical Fourier harmonics led to a high fit index, always greater than $99.5 \%$, when the original outline was compared to the corresponding outline of the reconstructed sum of harmonics. However, the major anatomical parts of the mandible were described by the 10 first harmonics (Figure 2; Table 1). Likewise the discriminant analysis of the elliptical descriptors was performed for the first 10 harmonics because the multivariate analysis was no more significant after these harmonics (Wilks' Lambda values $p>0.05)$.

Table 1 | Elliptical Fourier analysis of the lateral outline of the mandible in WT, Eda ${ }^{\mathrm{Ta} / \mathrm{Y}}$, and $E d a^{\mathrm{T} /+}$ mice. Summary statistics of the elliptical descriptors of the first 10 harmonics.

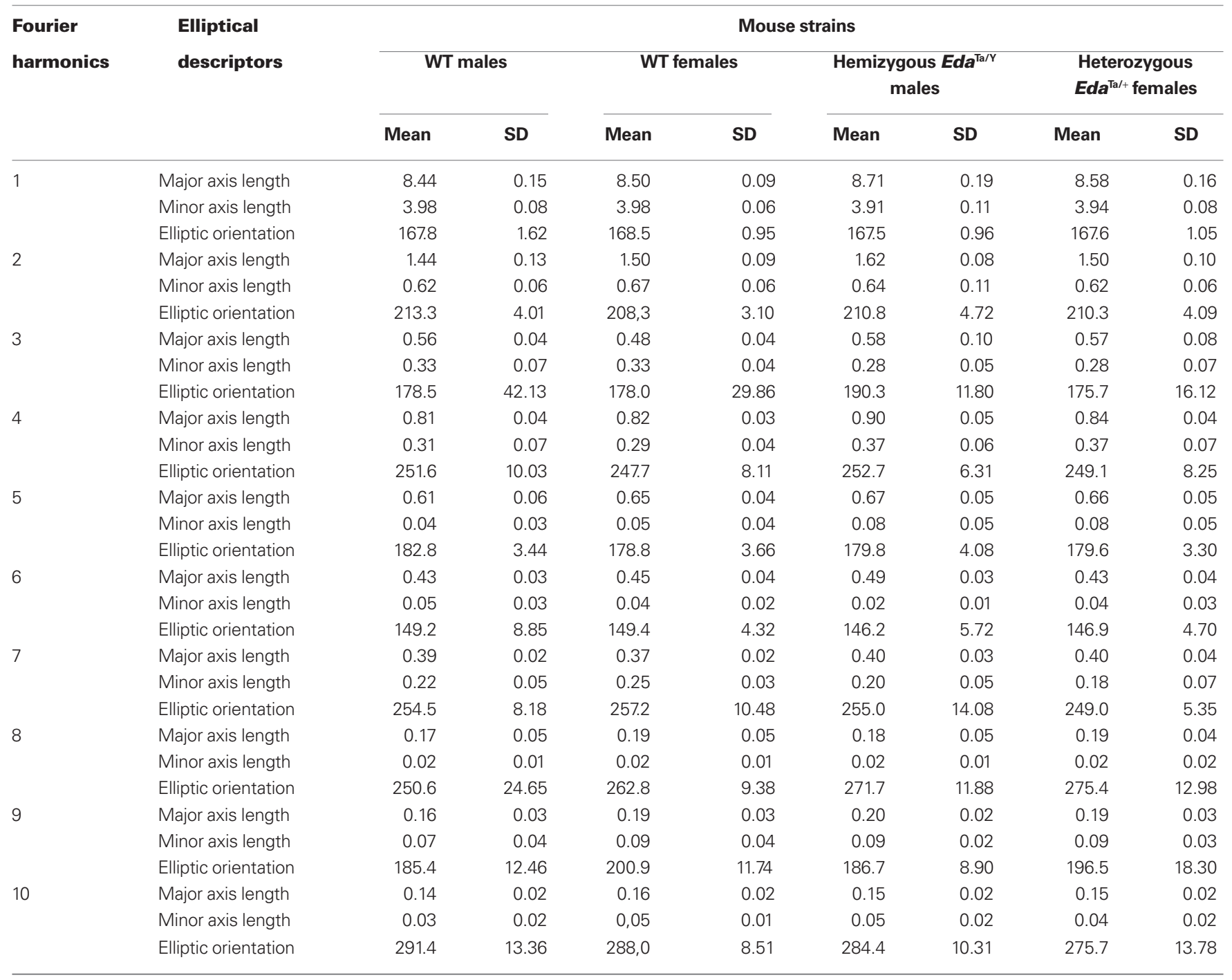

Elliptic orientation is given in degree and corresponds to the angle between the major axis of the ellipse and the horizontal axis of reference. SD, standard deviation. 
Subtle but significant mandibular shape differences were demonstrated between hemizygous $E d a^{\mathrm{T} a / Y}$ specimens, WT males, and a group formed by heterozygous $E d a^{\mathrm{T} a /+}$ females and WT females (Figure 4). However, the discriminant analysis did not reveal significant differences between $E d a^{\mathrm{Ta} /+}$ females and WT females. The first two discriminant functions (DF1, DF2) described $94.6 \%$ of the total variance, the first accounting for $60.6 \%$ of the total variance, the second DF for the remaining $34.0 \%$.

Hemizygous Ta males clearly departed from the other mouse groups as shown by the significant distances separating the $E d a^{\mathrm{Ta} / \mathrm{Y}}$ specimens from the other mice groups (Figure 4) and by the accuracy of the classification (see Table 2 ). The greatest difference occurred between hemizygous Ta males and WT males, and the lower distinction between Ta males and heterozygous Ta females, as demonstrated by the corresponding inter-group distances (Figure 4). The variables that were most effective in the distinction of hemizygous Ta specimens from the WT males sample along DF1 were the major axis length of the first, second, fourth, and ninth harmonics (Table 3). DF2, accounting for the distinction between hemizygous $E d a^{\mathrm{Ta} / \mathrm{Y}}$ and heterozygous $E d a^{\mathrm{Ta} /+}$ mice, was best correlated with the major axis length of the fourth Fourier ellipse and the orientation of the ninth ellipse (Table 3 ).

A noticeable overlap occurred between heterozygous $E d a^{\mathrm{Ta} /+}$ females and WT females (Figure 4). The corresponding betweengroup distance was not significant and the group classification was also less accurate (Table 2). This overlap indicated a common pattern of mandibular morphology within the two mouse strains.

In terms of mandibular shape variations, the morphological differentiation between hemizygous Ta males and the WT males consisted of geometrical differences in the first, second, fourth, and ninth Fourier ellipses mainly (Table 3 ). The more elongated first ellipse in hemizygous Ta males reflected a shorter mandibular

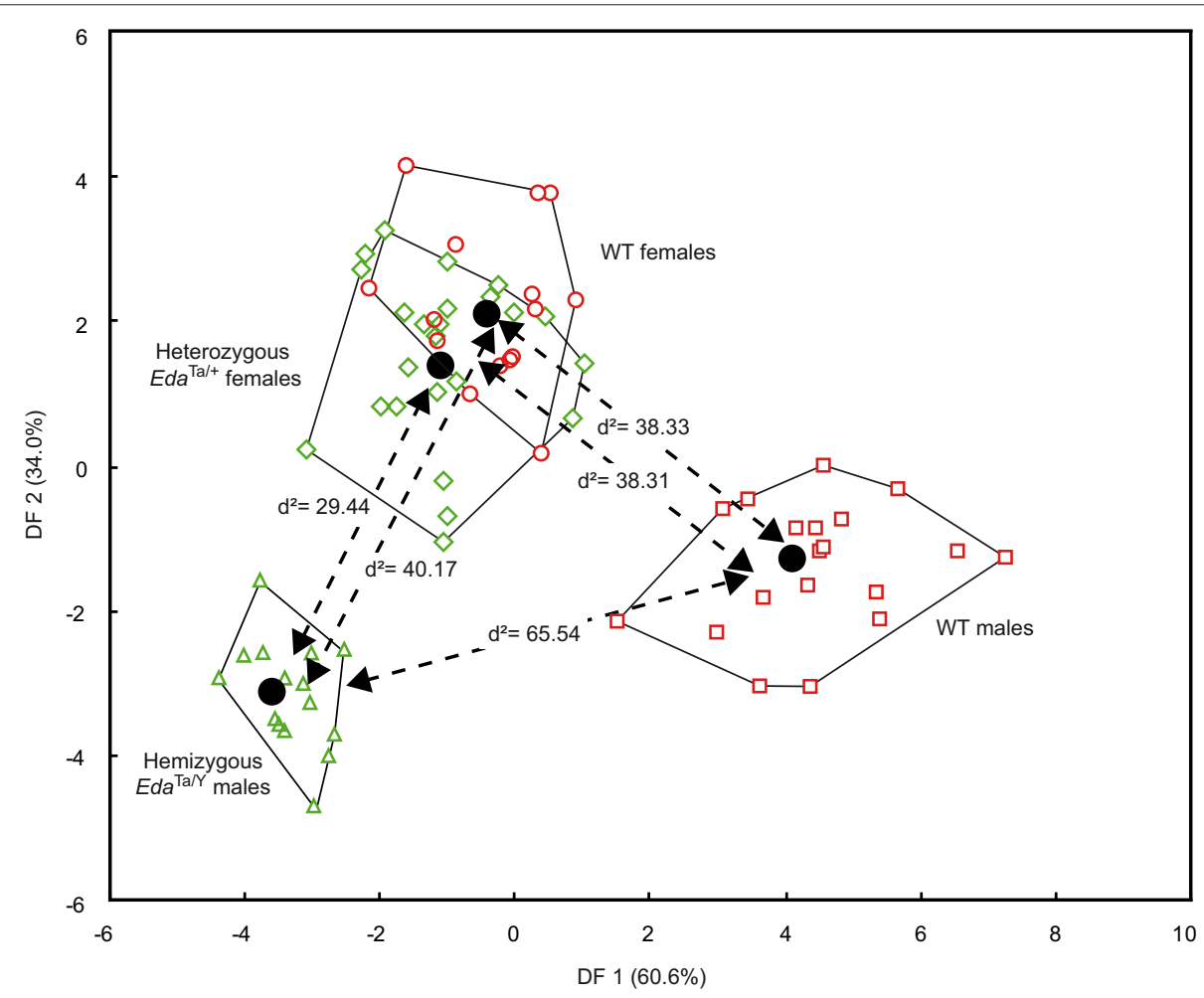

FIGURE 4 | Results of the discriminant analysis of the mandibular elliptical Fourier descriptors. Scatterplots of the Wild-type (males: red squares; females: red circles), hemizygous Eda ${ }^{\mathrm{Ta} /}$ (green triangles), and heterozygous Eda ${ }^{\mathrm{Ta} /+}$ (green diamonds) specimens along the first and second canonical discriminant axes (DF1, DF2). The unbiased Mahalanobis $D^{2}$ distances between the centroids of each mouse strain group are reported.

Table 2 | Results of the classification procedure and cross-validation procedure.

Mouse strains

\begin{tabular}{|c|c|c|c|c|}
\hline & \\
\hline & WT females & WT males & Heterozygous $\mathrm{Eda}^{\mathrm{Ta} / \mathrm{f}}$ females & Hemizygous $\boldsymbol{E d a}^{\mathrm{Ta} / \mathrm{Y}}$ males \\
\hline WT females & $87.5 / 62.5$ & $0.0 / 6.2$ & $12.5 / 31.3$ & $0.0 / 0.0$ \\
\hline WT males & $0.0 / 5.3$ & $100.0 / 89.5$ & $0.0 / 5.2$ & $0.0 / 0.0$ \\
\hline Heterozygous Eda ${ }^{\top a /+}$ females & $12.5 / 33.3$ & $0.0 / 4.2$ & $87.5 / 50.0$ & $0.0 / 12.5$ \\
\hline Hemizygous Eda ${ }^{T a /}$ males & $0.0 / 0.0$ & $0.0 / 0.0$ & $0.0 / 0.0$ & $100.0 / 100.0$ \\
\hline
\end{tabular}




\begin{tabular}{|c|c|c|c|c|c|c|}
\hline \multirow{2}{*}{$\begin{array}{l}\text { Fourier } \\
\text { harmonics }\end{array}$} & \multicolumn{3}{|c|}{ First discriminant axis } & \multicolumn{3}{|c|}{ Second discriminant axis } \\
\hline & $\begin{array}{l}\text { Major axis } \\
\text { length }\end{array}$ & $\begin{array}{l}\text { Minor axis } \\
\text { length }\end{array}$ & $\begin{array}{l}\text { Elliptic } \\
\text { orientation }\end{array}$ & $\begin{array}{l}\text { Major axis } \\
\text { length }\end{array}$ & $\begin{array}{l}\text { Minor axis } \\
\text { length }\end{array}$ & $\begin{array}{l}\text { Elliptic } \\
\text { orientation }\end{array}$ \\
\hline 2 & $-0.22^{*}$ & -0.04 & 0.10 & -0.12 & 0.05 & -0.13 \\
\hline 3 & -0.02 & 0.11 & -0.03 & -0.14 & 0.01 & -0.07 \\
\hline 4 & $-0.28^{*}$ & -0.13 & 0.01 & $-0.22^{*}$ & -0.07 & -0.10 \\
\hline 5 & -0.17 & -0.10 & 0.12 & 0.02 & -0.02 & -0.10 \\
\hline 6 & -0.13 & 0.14 & 0.06 & -0.15 & 0.07 & 0.03 \\
\hline 10 & -0.04 & -0.18 & 0.11 & 0.09 & 0.03 & -0.09 \\
\hline
\end{tabular}

$r$, Coefficient of correlation; * indicates correlation coefficient $\geq 0.20$.

ramus, and the greater major axis length of the second and fourth ellipses characterized a lower mandibular corpus (Figure 2). The aspect of the coronoid process in hemizygous Ta males seemed also to be different; the greater horizontal elongation of the ninth harmonic reflected a marked backward projection of this structure (Figure 5). The contributions of the Fourier ellipse from the sixth harmonic could be characterized by a slightly narrower neck of the mandibular condyle in Ta males compared to WT males (Figure 6).

Besides, hemizygous Ta males departed from the heterozygous Ta females-WT females cluster in having, on average, a lower mandibular corpus as demonstrated by the greater major axis length of the fourth ellipse in $E d a^{\mathrm{T} / \mathrm{Y}}$ specimens (Tables 1 and 3). Nevertheless some heterozygous Ta females seemed to present a relative morphological proximity with hemizygous Ta males; along DF2, some specimens falling near the Ta males group (Figure 4).

The overall size of the mandible differed between males and females in both WT and Ta mice (Figure 7). Welch $F$ test showed significant differences between mouse groups $(F=19.25, p<0.001)$. In the WT sample, females presented larger mandibles than males. Ta mice also evidenced significant size differences, and the magnitude of the size differences paralleled those observed within the WT mouse sample. No statistical differences were observed between $E d a^{T / Y}$ specimens and WT males. However, Ta mice evidenced a higher mandibular size variability with $E d a^{\mathrm{T} / \mathrm{Y}}$ exhibiting the greatest range of variation.

\section{DISCUSSION}

Elliptical Fourier analysis treats the shape of the mandible as a whole (Kuhl and Giardana, 1982; Schmittbuhl et al., 2003) contrary to other morphometric approaches based on anatomical landmarks and their associated measurements (Richtsmeier et al., 2002). The few studies that investigated the mandibular change in $\mathrm{Ta} /$ eda mice provide only general descriptions, based on a visual estimation of the mandibular abnormalities (Grüneberg, 1971).
To our knowledge no quantitative analysis of the mandibular phenotype has been undertaken although the skull and facial bones are dysmorphic in the Ta mutant and in human embryos with XLHED (Montonen et al., 1998). Elliptical Fourier analysis of the mandibular profile provides for the first time quantitative evidence of significant mandibular differences between mice carrying the $E d a / T a$ gene mutation and their non-mutant littermates.

The mandibular profile alone enables a clear distinction between $E d a^{\mathrm{T} / \mathrm{Y}}$ group and the other mouse groups. Indeed, our results do not reveal any significant mandibular size differences between hemizygous Ta males and their corresponding WT specimens (Figure 7). Hence, the mandible is another key feature for identifying the effects of $E d a / T a$ mutations, and the distinctiveness of hemizygous Ta mice suggests that Eda/Ta defects lead mainly to shape abnormalities rather than size discrepancies of the mandible. Even if a relationship between sex and mandibular shape within the mouse strains exists (see the differences between males and females in WT sample, Figure 3), the marked departure of $E d a^{\mathrm{Ta} / \mathrm{Y}}$ specimens from WT males compared to the lower distinction between hemizygous $E d a^{\mathrm{Ta} / \mathrm{Y}}$ males and heterozygous $E d a^{\mathrm{Ta} /+}$ females provides evidence for a predominant effect of the Ta mutation on the mandibular morphology.

The marked morphological distinction of the mandibular features in hemizygous $E d a^{\mathrm{T} / \mathrm{Y}}$ mice integrated the well-known severe phenotypic expression observed in mutant Ta males (Sofaer, 1969; Blecher, 1986). Such result parallels previously well-established skeletal abnormalities such as abnormal tail vertebra (Hill et al., 2002). Even though these bones develop from mesenchymal condensation (Dixon, 1997), the osseous dysmorphologies found in hemizygous Ta mice emphasize the role of EDA-A in the reciprocal epithelial-mesenchymal interactions that are of critical importance in the normal formation of the skeleton (Hall, 1980; Hall and Coffin-Collins, 1990; Dixon, 1997), and suggest that EDA-A expression in osteoblast may be a functional important factor in bone development (Hill et al., 2002). 


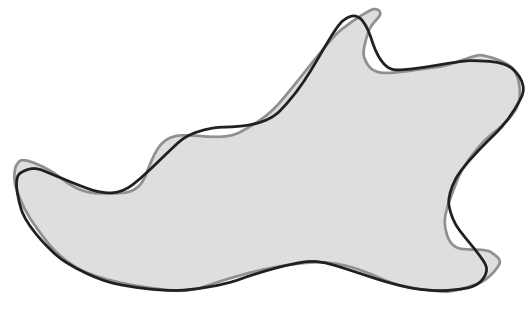

1 to 8

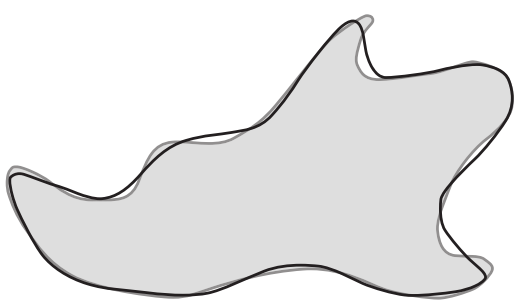

1 to 9

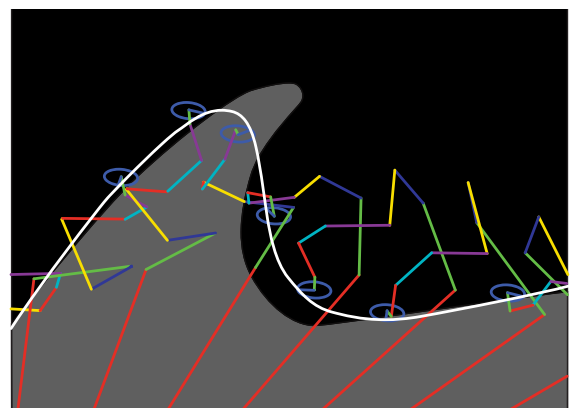

WT
specimen

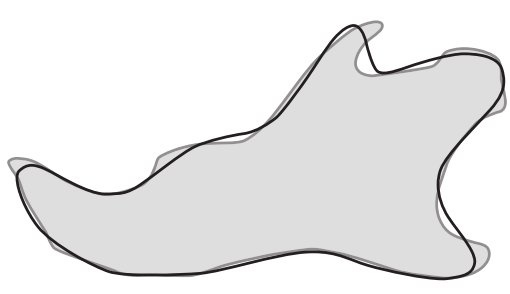

1 to 8

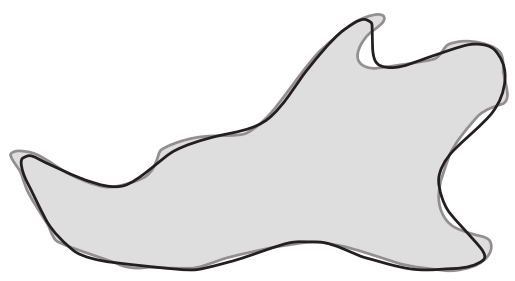

1 to 9

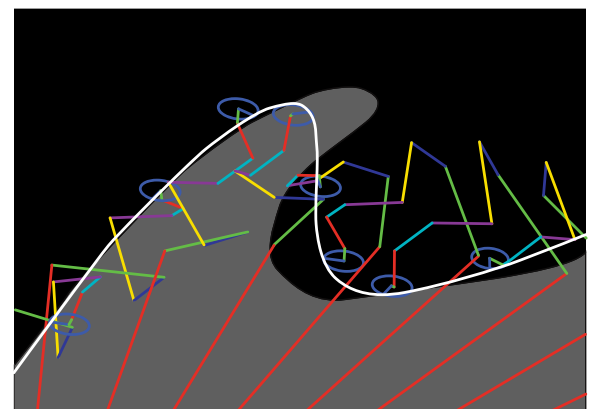

Hemizygous Tabby

specimen

FIGURE 5 | Subtle shape differences between WT and hemizygous Tabby specimens highlighted by the contribution of the ninth Fourier ellipse. The greater elongation of the ninth ellipse (blue) reflects a more backward projection of the coronoid process in the hemizygous Tabby specimen.
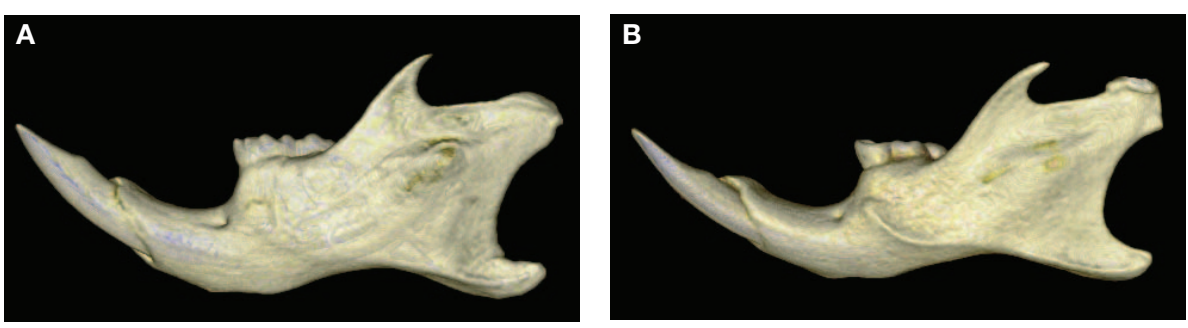

FIGURE 6 | Morphological comparison of the mandibular morphology between WT (A) and hemizygous Tabby (B) specimens. The 3D reconstructions illustrate the marked backward projection of the coronoid process, the narrower neck of the condyle, and the lower corpus of the mandible in the hemizygous Tabby specimen.

Abnormalities of mandible in hemizygous Ta mice mainly consisted in a restrained development of that bone along dorsoventral axis, the height of the mandibular corpus being particularly affected. Subtle shape changes also occur, corresponding to a marked backward projection of the coronoid process and to a narrowing of the mandibular condylar neck (Figures 5 and 6). The mechanisms responsible for these subtle morphological changes remain unclear. Our results have to be supported by further investigations in order to precise whether these changes correspond to compensations for other craniofacial dysmorphologies - e.g., the reduced and retrognatic maxilla-observed in XLHED patients (Bondarets et al., 2002; Johnson et al., 2002; Dellavia et al., 2006) but not yet demonstrated in mutant Ta mice or are caused directly by the mutation itself. However, the noticeable mandibular changes parallel the marked abnormalities of the lower tooth row (Kristenova et al., 2002; Peterkova et al., 2002). Hemizygous Ta specimens, in exhibiting 


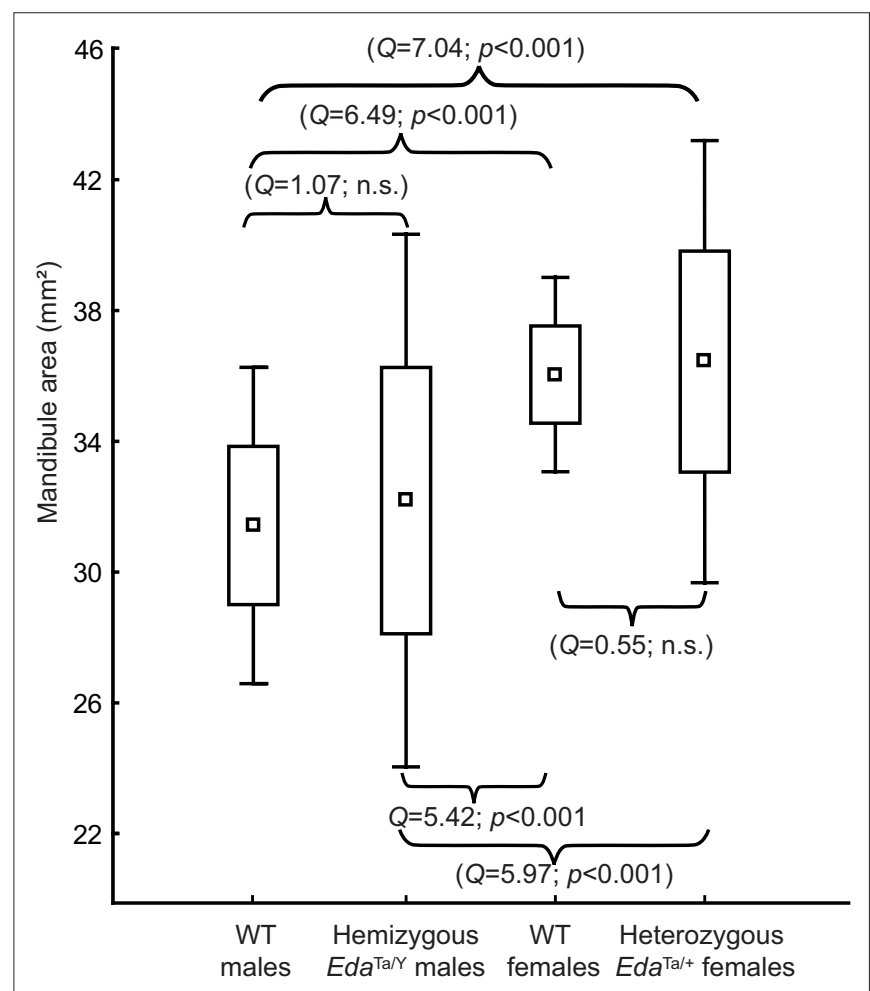

FIGURE 7 | Size variation of the mandible in wild-type, hemizygous $E \boldsymbol{d a} \boldsymbol{a}^{\mathrm{Ta} / \mathrm{y}}$ and heterozygous $\mathbf{E d a ^ { \mathrm { Ta } / \mathrm { y } }}$ mice. Comparable magnitude of size differences were depicted between WT males-WT females and between hemizygous Tabby males-heterozygous Tabby females. Results of the post hoc pairwise between-group comparisons (Tukey's test) are reported. Box indicates mean (square) $\pm 1 \mathrm{SD}$; outliers correspond to minimum and maximum mandibular areas.

very distinct pattern of dental and mandibular differentiation from WT specimens, provide thus further evidence that the mandibular dysmorphologies in $E d a^{\mathrm{T} a / Y}$ mice are strongly correlated with $E d a / T a$ gene defects.

Heterozygous Ta mice seem to be less affected regarding mandibular shape, as suggested by the important overlap occurring with WT females (Figure 3). Nevertheless, shape variation seems to be higher in heterozygous females than in hemizygous males, some specimens presenting a relative morphological proximity with hemizygous Ta mice (Figure 3). This pattern of variation is congruent with the marked degree of tooth row polymorphism observed in heterozygous $E d a^{\mathrm{Ta} /+}$ females (Sofaer, 1969; Kristenova et al., 2002; Peterkova et al., 2002; Charles et al., 2009). The higher variability of tooth and mandibular morphology might be consistent with a probable X-inactivation effect in females (Lyon, 1961; Plath et al., 2002). This molecular mechanism leads, in heterozygous Ta females, to a mosaic of cells with expression of a wild-type or a null

\section{REFERENCES}

Arslan, S., Devecioğlu Kama, J., Ozer, T., and Yavuz, I. (2007). Craniofacial and upper airway cephalometrics in hypohidrotic ectodermal dysplasia. Dentomaxillofac. Radiol. 36, 478-483.
Blecher, S. R. (1986). Anhidrosis and absence of sweat glands in mice hemizygous for the tabby gene: supportive evidence for the hypothesis of homology between tabby and human anhidrotic (hypohidrotic) ectodermal dysplasia (Christ-Siemens-Touraine

Eda gene. The X-inactivation effect can thus induce various pattern of dental and mandibular development, explaining the higher morphological variability recorded in heterozygous Ta females.

The underlying molecular aspects of the mandibular dysmorphologies could be linked, first of all, to a direct role of Eda in the development of the craniofacial complex (Montonen et al., 1998; Bushdid et al., 2001), and especially in Meckel's cartilage (Tucker et al., 2000). But the function of EDA in normal craniofacial development and in craniofacial abnormalities found in HED is still relatively elusive.

A hypothesis could be that morphological alteration in Ta mice mandible may be a secondary effect due to defective ectodermal development (Mina, 2001; Pummila et al., 2007). According to Haworth et al. (2004), cells that will occupy the epithelium of the distal and the proximal mandible primordium already occupy different spatial locations in the developing head ectoderm prior to the formation of the first pharyngeal arch and neural crest migration. Consequently, ectodermal abnormalities such as those linked to $E d a / T a$ mutations could be responsible to alterations of mandibular development. Besides, the involvement of some ectodermal genes in mandibular patterning has been emphasized as suggested for example by the inactivation of Ptx1, another ecto-mesodermal gene, leading to chondrogenesis alterations and to the loss of bones derived from the proximal part of the mandibular mesenchyme (Lanctôt et al., 1999).

Considering mandibular shape, 2D elliptical Fourier descriptors provides an original description of the distinctive mandibular manifestation in Ta mice. The ability of our quantitative approach for depicting subtle differences in mandibular profiles (e.g., marked backward projection of the coronoid process and narrowing of the mandibular condylar neck) eludes detection by conventional morphometric methods. The mandibular shape differences evidenced in this study parallel the tooth abnormalities and skeletal defects observed in Ta mice, underlining the role of EDA-A in the reciprocal epithelial-mesenchymal interactions that are of critical importance in the normal dental and craniofacial development. Even if more extensive studies should be undertaken to explore the whole craniofacial skeleton, we provide a detailed analysis of global and local mandibular dysmorphologies and identify the mandibular location that should be targeted for more detailed analysis of cell and molecular processes linked to $\mathrm{Eda} / \mathrm{Ta}$ gene mutations.

\section{ACKNOWLEDGMENTS}

We thank Dr. Y. Bolender for reviewing the English version.

\section{SUPPLEMENTARY MATERIAL}

The Supplementary Material for this article can be found online at http://www.frontiersinneuroscience.org/ craniofacial_biology/10.3389/fphys.2011.00015/abstract/

syndrome). J. Invest. Dermatol. 87, 720-722.

Bondarets, N., Jones, R. M., and McDonald, F. (2002). Analysis of facial growth in subjects with syndromic ectodermal dysplasia: a longitudinal analysis. Orthod. Craniofac. Res. 5, 71-84.
Bourgeois, P., Bolcato-Bellemin, A. L., Danse, J.M., Bloch-Zupan,A., Yoshiba, K., Stoetzel, C., and Perrin-Schmitt, F. (1998). The variable expressivity and incomplete penetrance of the twistnull heterozygous mouse phenotype resemble those of human Saethre- 
Chotzen syndrome. Hum. Mol. Genet. 7, 945-957.

Bushdid, P. B., Chen, C. L., Brantley, D. M., Yull, F., Raghow, R., Kerr, L. D., and Barnett,J.V. (2001).NF-kappaB mediates FGF signal regulation of msx-1 expression. Dev. Biol. 237, 107-115.

Cambiaghi, S., Restano, L., Pääkkönen, K., Caputo, R., and Kere, J. (2000). Clinical findings in mosaic carriers of hypohidrotic ectodermal dysplasia. Arch. Dermatol. 136, 217-224.

Charles, C., Pantalacci, S., Perterkova, R., Tafforeau, P., Laudet, V., and Viriot, L. (2009). Effect of eda loss of function on upper jugal tooth morphology. Anat. Rec. 292, 299-308.

Clauss, F., Maniere, M. C., Obry, F., Waltmann, E., Hadj-Rabia, S., Bodemer, C., Alembik, Y., Lesot, H., and Schmittbuhl, M. (2008). Dento-craniofacial phenotypes and underlying molecular mechanisms in hypohidrotic ectodermal dysplasia (HED): a review. J. Dent. Res. 87 , 1089-1099.

Dellavia, C., Catti, F., Sforza, C., Tommasi, D. G., and Ferrario, V. F. (2010). Craniofacial growth in ectodermal dysplasia. An 8 year longitudinal evaluation of Italian subjects. Angle Orthod. 80, 545-551.

Dellavia, C., Sforza, C., Malerba, A., Strohmenger, L., and Ferrario, V. F. (2006). Palatal size and shape in 6-year olds affected by hypohidrotic ectodermal dysplasia. Angle Orthod. 76, 978-983.

DiGiovanna, J. J., Priolo, M., and Itin, P. (2009). Approach towards a new classification for ectodermal dysplasias: integration of the clinical and molecular knowledge. Am. J. Med. Genet. A 149, 2068-2070.

Dixon, A. D. (1997). "Prenatal development of the facial skeleton," in Fundamentals of Craniofacial Growth, eds A. D. Dixon, D. A. N Hoyte, and O. Rönning (Boca Raton: CRC Press), 59-97.

Everitt, B., and Rabe-Hesketh, S. (2001). Analysis of Medical Data Using S-Plus. New York: Springer.

Grüneberg, H. (1971). The glandular aspects of the tabby syndrome in the mouse. J. Embryol. Exp. Morphol. 25, 1-19.

Hall, B. K. (1980). Tissue interactions and the initiation of osteogenesis and chondrogenesis in the neural crest-derived mandibular skeleton of the embryonic mouse as seen in isolated murine tissues and in recombinations of murine and avian tissues. J. Embryol. Exp. Morphol. 58, 251-264.

Hall, B.K., and Coffin-Collins, P.A. (1990). Reciprocal interactions between epithelium, mesenchyme, and epidermal growth factor (EGF) in the regulation of mandibular mitotic activity in the embryonic chick. J. Craniofac. Genet. Dev. Biol. 10, 241-261.

Hammer, O., Harper, D. A. T., and Ryan, P. D. (2001). PAST: paleontological statistics software package for education and data analysis. Palaeontol. Electronica 4, 1-9.

Haworth, K.E., Healy, C., Morgan, P., and Sharpe, P.T. (2004). Regionalisation of early head ectoderm is regulated by endoderm and prepatterns the orofacial epithelium. Development 131, 4797-4806.

Hill, N. L., Laib, A., and Duncan, M. K. (2002). Mutation of the ectodysplasinA gene results in bone defects in mice. J. Comp. Pathol. 126, 220-225.

Itin, P. H. (2009). Rationale and background as basis for a new classification of the ectodermal dysplasias. Am. J. Med. Genet. A 149, 1973-1976.

Johnson, E. L., Roberts, M. W., Guckes, A. D., Bailey, L. J., Phillips, C. L., and Wright, J.T. (2002). Analysis of craniofacial development in children with hypohidrotic ectodermal dysplasia. Am. J. Med. Genet. 112, 327-334.

Kristenova, P., Peterka, M., Lisi, S., Gendrault, J. L., Lesot, H., and Peterkova, R. (2002). Different morphotypes of functional dentition in the lower molar region of tabby (EDA) mice. Orthod. Craniofac. Res. 5, 205-214.

Kuhl, F. P., and Giardana, C. R. (1982) Elliptic Fourier features of closed contour. Comput. Graph. Image Process. $18,236-258$

Lanctôt, C., Moreau, A., Chamberland, M., Tremblay, M.L., and Drouin, J. (1999). Hindlimb patterning and mandible development require the Ptxl gene. Development 126,1805-1810.

Lesot, H., Clauss, F., Manière, M. C., and Schmittbuhl,M. (2009). Consequences of X-linked hypohidrotic ectodermal dysplasia for the human jaw bone. Front. Oral Biol. 13, 93-99.

Lyon, M. (1961). Gene action in the $\mathrm{X}$-chromosome of the mouse (Mus musculus L.). Nature 190, 372-373.

Mina, M. (2001). Regulation of mandibular growth and morphogenesis. Crit. Rev. Oral Biol. Med. 12, 276-300.

Monreal,A.W.,Zonana, J., and Ferguson, B. (1998). Identification of a new splice form of the EDA1 gene permits detection of nearly all X-linked hypohidrotic ectodermal dysplasia mutations. Am. J. Hum. Genet. 63, 380-389.

Montonen, O., Ezer, S., Saarialho-Kere, U. K., Herva, R., Karjalainen-Lindsberg, M. L., Kaitila, I., Schlessinger, D., Srivastava, A. K., Thesleff, I., and Kere, J. (1998). The gene defective in anhidrotic ectodermal dysplasia is expressed in the developing epithelium, neuroectoderm, thymus, and bone. $J$. Histochem. Cytochem. 46, 281-289.

Peterkova, R., Kristenova, P., Lesot, H., Lisi, S., Vonesch, J. L., Gendrault, J. L. and Peterka, M. (2002). Different morphotypes of the tabby (EDA) dentition in the mouse mandible result from a defect in the mesio-distal segmentation of dental epithelium. Orthod. Craniofac. Res. 5, 215-226.

Pinheiro, M., and Freire-Maia, N. (1994). Ectodermal dysplasias: a clinical classification and a causal review. Am. J. Med. Genet. 53, 153-162.

Plath, K., Mlynarczyk-Evans, S., Nusinow, D., and Panning, B. (2002). XIST RNA and the mechanism of X chromosome inactivation. Annu. Rev. Genet. 36, 233-278.

Pummila, M., Fliniaux, I., Jaatinen, R. James, M. J., Laurikkala, J., Schneider, P., Thesleff, I., and Mikkola, M. L. (2007).Ectodysplasin has a dual role in ectodermal organogenesis: inhibition of Bmp activity and induction of Shh expression. Development 134,117-125.

Richtsmeier, J., Zumwalt, A., Carlson, E., Epstein, C., and Reeves, R. (2002). Craniofacial phenotypes in segmentally trisomic mouse models for Down syndrome. Am. J. Med. Genet. 107, 317-324.

Schmittbuhl, M., Allenbach, B., Le Minor, J. M., and Schaaf, A. (2003). Elliptical descriptors: some simplified morphometric parameters for the quantification of complex outlines. Math. Geol. $35,853-872$.
Sofaer, J. A. (1969). Aspects of the tabbycrinkled and downless syndrome. I. The development of tabby teeth. $J$. Embryol. Exp. Morphol. 22, 181-205.

Srivastava, A. K., Pispa, J., Hartung, A. J., Du, Y., Ezer, S., Jenks, T., Shimada T., Pekkanen, M., Mikkola, M. L., Ko, M. S., Thesleff, I., Kere, J., and Schlessinger, D. (1997). The tabby phenotype is caused by mutation in a mouse homologue of the EDA gene that reveals novel mouse and human exons and encodes a protein (ectodysplasin-A) with collagenous domains. Proc. Natl. Acad. Sci. U.S.A. 94, 13069-13074.

Tucker, A. S., Headon, D. J., Schneider P., Ferguson, B. M., Overbeek, P., Tschopp, J., and Sharpe, P. T. (2000). Edar/Eda interactions regulate enamel knot formation in tooth morphogenesis. Development 127, 4691-4700.

Vincent, M. C., Biancalana, V., Ginisty, D., Mandel, J. L., and Calvas, P. (2001) Mutational spectrum of the ED1 gene in X-linked hypohidrotic ectodermal dysplasia. Eur. J. Hum. Genet. 9, 355-363.

Conflict of Interest Statement: The authors declare that the research was conducted in the absence of any commercial or financial relationships that could be construed as a potential conflict of interest.

Received: 20 February 2011; accepted: 26 March 2011; published online: 20 April 2011.

Citation: Bornert F, Choquet P, Gros CI, Aubertin G, Perrin-Schmitt F, Clauss F, Lesot H, Constantinesco A and Schmittbuhl $M$ (2011) Subtle morphological changes in the mandible of Tabby mice revealed by micro-CT imaging and elliptical Fourier quantification. Front. Physio. 2:15. doi: 10.3389/fphys.2011.00015

This article was submitted to Frontiers in Craniofacial Biology, a specialty of Frontiers in Physiology.

Copyright $\odot 2011$ Bornert, Choquet, Gros, Aubertin, Perrin-Schmitt, Clauss, Lesot, Constantinesco and Schmittbuhl. This is an open-access article subject to a nonexclusive license between the authors and Frontiers Media SA, which permits use, distribution and reproduction in other forums, provided the original authors and source are credited and other Frontiers conditions are complied with. 